Supplementary Information

\title{
Ultrafast Carrier Dynamics in Methylammonium Lead Bromide Perovskite
}

\author{
Xiaofan Deng ${ }^{a}$, Xiaoming Wen $^{a}{ }^{*}$, Shujuan Huang ${ }^{a}$, Rui Sheng ${ }^{a}$, Takaaki Harada ${ }^{b}$, \\ Tak W. Kee ${ }^{b}$, Martin Green ${ }^{a}$, and Anita Ho-Baillie ${ }^{a}$
}

a Australian Centre for Advanced Photovoltaics, The University of New South Wales, Sydney, 2052, Australia

${ }^{\mathrm{b}}$ Department of Chemistry, The University of Adelaide, Adelaide, 5005, Australia.

\section{Calculation method of carrier injection level}

The fs-TAS experiments are performed with fixed pump pulse wavelength of 400 $\mathrm{nm}$ and various pump fluence from 2 to $100 \mu \mathrm{J} / \mathrm{cm}^{2} /$ pulse. The pump fluence essentially determines the carrier injection level $\left(\mathrm{n}_{0}\right)$. Converting pump fluence to $\mathrm{n}_{0}$ is useful for comparing carrier dynamics data between different experiments even with different technologies. Similar to previous work ${ }^{1}$, Equation (S1) is the function used to estimate $\mathrm{n}_{0}$ from pump fluence:

$$
n_{0}=\frac{A(\lambda) \times \text { pump fluence }}{E_{p h}(\lambda) \times w}
$$

where $\lambda=400 \mathrm{~nm}$ is the wavelength of pump is pulse; $E_{p h}(400 \mathrm{~nm})=4.97 \times$ $10^{-13} \mu \mathrm{J}$ is the photon energy in pump; $w \approx 450 \mathrm{~nm}$ is thickness (determined by 
SEM) of perovskite film used in this experiment and $A(400 \mathrm{~nm}) \approx 0.94$ is the absorption of $400 \mathrm{~nm}$ pump in perovskite film. $A(400 \mathrm{~nm})$ is calculated by Equation (S2):

$$
A(\lambda)=1-e^{-\alpha(\lambda) * w}
$$

The $\alpha(\lambda)$ is the absorption coefficient at wavelength of $\lambda$, as shown in Figure 1(a) in main text. Therefor the injection level corresponding to each pump fluence is displayed in Table S1.

Table S1: Estimated injection level corresponding to each pump fluence.

\begin{tabular}{|c|c|c|c|c|}
\hline Pump Flunece $\left(\mu \mathrm{J} / \mathrm{cm}^{2}\right)$ & 2 & 10 & 50 & 100 \\
\hline Injection Level $\left(\mathrm{cm}^{-3}\right)$ & $7.60 \mathrm{E}+16$ & $3.80 \mathrm{E}+17$ & $1.90 \mathrm{E}+18$ & $3.80 \mathrm{E}+18$ \\
\hline
\end{tabular}

\section{Error analysis of time constants fitting}

The time constants fitting results with uncertainties and R-square values are shown in Table 2. The R-square value of each fitting is greater than 0.95 . Especially with high pump fluence $\left(50\right.$ and $100 \mathrm{uJ} / \mathrm{cm}^{2}$ ), due to the high signal/noise ratio the Rsquare values are larger than 0.995 which show the great reliability. With high pump fluence, all of the uncertainties of fitted time constants are smaller than $10 \%$. It suggests that the discussions and calculations based on these data are fairly reasonable. It should be noted that the uncertainties of $\tau_{3}$ are large that even more than $50 \%$ with low pump fluence because the values are beyond the experiment time window. Therefore, we do not make much discussion on $\tau_{3}$ in this work. 
Table 2: Exponential fitting results of carrier dynamics in $\mathrm{CH}_{3} \mathrm{NH}_{3} \mathrm{PbBr}_{3}$ sample at $534 \mathrm{~nm}$ with each pump fluence

\begin{tabular}{|c|c|c|c|c|c|c|c|c|c|}
\hline \multirow{2}{*}{\begin{tabular}{|c|} 
Pump \\
Fluence \\
$(\mathrm{uJ} / \mathrm{cm} 2)$
\end{tabular}} & \multicolumn{2}{|c|}{ Rise Process } & \multicolumn{3}{|c|}{ Fast Decay Component } & \multicolumn{3}{|c|}{ Slow Decay Component } & \multirow{2}{*}{$\mathrm{R}^{\wedge} 2$} \\
\hline & $\tau 1$ & $\Delta \tau 1$ & Fra & $\tau 2$ & $\Delta \tau 2$ & $\mathrm{Fr}$ & g) & $\Delta \tau 3$ & \\
\hline 2 & 0.271 & 0.020 & $20.0 \%$ & 248 & 71 & 80. & 3340. & 1790 & 0.969 \\
\hline 10 & 0.336 & 0.023 & $14.1 \%$ & 126 & 39 & $85.9 \%$ & 4470 & 1210 & 0.958 \\
\hline 50 & 0.472 & 0.034 & $46.7 \%$ & 86.4 & 5.4 & $53.3 \%$ & 1760 & 110 & 0.998 \\
\hline 100 & 0.469 & 0.045 & $48.7 \%$ & 75.3 & 7.3 & $51.3 \%$ & 1830 & 180 & 0.996 \\
\hline
\end{tabular}

\section{Reference}

1. Manser, J. S.; Kamat, P. V., Band Filling with Free Charge Carriers in Organometal Halide Perovskites. Nature Photonics 2014, 8, 737. 